\title{
Partitions of Natural Numbers with the Intersection Not Empty
}

\author{
Wen Yu and Min Tang \\ School of Mathematics and Computer Science, Anhui Normal University, Wuhu 241003, China \\ Correspondence should be addressed to Min Tang; tangmin@mail.ahnu.edu.cn
}

Received 14 May 2012; Accepted 19 July 2012

Academic Editors: A. Cossidente, M. Eliasi, L. Feng, and M. Gionfriddo

Copyright (c) 2013 W. Yu and M. Tang. This is an open access article distributed under the Creative Commons Attribution License, which permits unrestricted use, distribution, and reproduction in any medium, provided the original work is properly cited.

Let $\mathbb{N}$ be the set of nonnegative integers. For a given set $A \subset \mathbb{N}$ the representation functions $R_{2}(A, n), R_{3}(A, n)$ are defined as the number of solutions of the equation $n=a+a^{\prime}, a, a^{\prime} \in A$ with condition $a<a^{\prime}, a \leq a^{\prime}$, respectively. In this paper, we prove that if $\mathbb{N}=A \cup B$ and $A \cap B=\{8 k: k \in \mathbb{N}\}$, then $R_{i}(A, n)=R_{i}(B, n)$ cannot hold for all sufficiently large integers $n$ where $i=2,3$.

\section{Introduction}

Let $\mathbb{N}$ be the set of nonnegative integers. For $A \subset \mathbb{N}$, let $R_{1}(A, n), R_{2}(A, n), R_{3}(A, n)$ denote the number of solutions of

$$
\begin{gathered}
a+a^{\prime}=n, \quad a, a^{\prime} \in A, \\
a+a^{\prime}=n, \quad a, a^{\prime} \in A, a<a^{\prime}, \\
a+a^{\prime}=n, \quad a, a^{\prime} \in A, a<a^{\prime},
\end{gathered}
$$

respectively. Sárközy asked ever whether there exist two sets $A$ and $B$ of nonnegative integers with infinite symmetric difference, that is:

$$
\begin{gathered}
|(A \cup B) \backslash(A \cap B)|=\infty, \\
R_{i}(A, n)=R_{i}(B, n), \quad n \geq n_{0}
\end{gathered}
$$

for $i=1,2,3$. As Dombi [1] has shown, the answer is negative for $i=1$ by the simple observation that $R_{1}(A, n)$ is odd if and only if $n=2 a$ for some $a \in A$. For $i=2$, Dombi presented a partition of the set of all positive integers into two disjoint subsets $A$ and $B$ such that $R_{2}(A, n)=R_{2}(B, n)$ for all sufficiently large $n$. For $i=3$, Chen and Wang [2] constructed sets $A, B$ such that $R_{3}(A, n)=R_{3}(B, n)$ for all integers $n \geq 3$, where $A$ is the set of all those positive integers $n$ such that the number of zeroes in the binary representation of $n-1$ is even, and $B$ is the set of all those positive integers $n$ such that the number of zeroes in the binary representation of $n-1$ is odd. For the other related results, the reader is referred to see [3-7].
It is natural to ask the following: for $i=2,3$, are there subsets $A, B \subset \mathbb{N}$ with $R_{i}(A, n)=R_{i}(B, n)$ for all large enough integers $n$ such that $\mathbb{N}=A \cup B$ and $A \cap B \neq \varnothing$ ? Recently, the authors of this paper [8] obtained some results in this direction.

In this paper, we obtain the following results.

Theorem 1. If $\mathbb{N}=A \cup B$ and $A \cap B=\{8 k: k \in \mathbb{N}\}$, then $R_{2}(A, n)=R_{2}(B, n)$ cannot hold for all sufficiently large integers $n$.

Theorem 2. If $\mathbb{N}=A \cup B$ and $A \cap B=\{8 k: k \in \mathbb{N}\}$, then $R_{3}(A, n)=R_{3}(B, n)$ cannot hold for all sufficiently large integers $n$.

Remark 3. For $r=1,2,3,4,5,6,7$, similarly, we can prove that if $\mathbb{N}=A \cup B$ and $A \cap B=\{8 k+r: k \in \mathbb{N}\}$, then $R_{i}(A, n)=$ $R_{i}(B, n)$ cannot hold for all sufficiently large integers $n$, where $i=2,3$.

\section{Proof of Theorem 1}

Proof. Suppose that there exist integer $n_{0}$ and sets $A, B \subset \mathbb{N}$ with $A \cup B=\mathbb{N}$ and $A \cap B=\{8 k: k \in \mathbb{N}\}$ such that $R_{2}(A, n)=$ $R_{2}(B, n)$ for all $n \geq n_{0}$. Without loss of generality, we may assume that $n_{0}=16 N-1, N$ is a positive integer; then there exists a polynomial $p(x)$ of degree at most $16 N-2$ such that

$$
\sum_{n=0}^{\infty}\left(R_{2}(A, n)-R_{2}(B, n)\right) x^{n}=p(x) .
$$


Let

$$
\begin{gathered}
f(x)=\sum_{a \in A} x^{a}, \quad g(x)=\sum_{b \in B} x^{b}, \\
\varepsilon_{i}= \begin{cases}1, & i \in A, \\
0, & i \notin A .\end{cases}
\end{gathered}
$$

Then we have

$$
\begin{gathered}
f(x)=\sum_{a \in A} x^{a}=\sum_{i=0}^{\infty} \varepsilon_{i} x^{i} \\
\sum_{n=0}^{\infty} R_{2}(A, n) x^{n}=\frac{1}{2}\left(f^{2}(x)-f\left(x^{2}\right)\right) .
\end{gathered}
$$

Moreover,

$$
\begin{aligned}
& g(x)=\sum_{b \in B} x^{b}=\sum_{b \in(\mathbb{N} \backslash A) \cup(A \cap B)} x^{b} \\
& =\sum_{n=0}^{\infty} x^{n}-f(x)+\sum_{k=0}^{\infty} x^{8 k} \\
& =\frac{1}{1-x}+\frac{1}{1-x^{8}}-f(x) \text {, } \\
& \sum_{n=0}^{\infty} R_{2}(B, n) x^{n}=\frac{1}{2}\left(g^{2}(x)-g\left(x^{2}\right)\right) \\
& =\frac{1}{2}\left(\left(\frac{1}{1-x}+\frac{1}{1-x^{8}}-f(x)\right)^{2}\right. \\
& \left.-\left(\frac{1}{1-x^{2}}+\frac{1}{1-x^{16}}-f\left(x^{2}\right)\right)\right) \\
& =\frac{x}{(1-x)\left(1-x^{2}\right)}+\frac{x^{8}}{\left(1-x^{8}\right)\left(1-x^{16}\right)} \\
& +\frac{1}{(1-x)\left(1-x^{8}\right)}-\frac{f(x)}{1-x}-\frac{f(x)}{1-x^{8}} \\
& +\frac{1}{2} f^{2}(x)+\frac{1}{2} f\left(x^{2}\right) \text {. }
\end{aligned}
$$

Therefore,

$$
\begin{aligned}
\sum_{n=0}^{\infty}\left(R_{2}(A, n)-R_{2}(B, n)\right) x^{n} \\
=\frac{f(x)}{1-x}+\frac{f(x)}{1-x^{8}}-f\left(x^{2}\right)-\frac{x}{(1-x)\left(1-x^{2}\right)} \\
\quad-\frac{x^{8}}{\left(1-x^{8}\right)\left(1-x^{16}\right)}-\frac{1}{(1-x)\left(1-x^{8}\right)} .
\end{aligned}
$$

Write

$$
p(x)\left(1-x^{8}\right)=\sum_{i=0}^{16 N+6} \alpha_{i} x^{i}
$$

By (3) and (7), we have

$$
\begin{aligned}
\left(\sum_{i=0}^{7} x^{i}+1\right) f(x) & \\
= & \left(1-x^{8}\right) f\left(x^{2}\right)+\frac{1+x+x^{3}+x^{5}+x^{7}}{1-x} \\
& +\frac{x^{8}}{1-x^{16}}+p(x)\left(1-x^{8}\right) .
\end{aligned}
$$

Writing both sides of (9) in power series, then we have

$$
\begin{aligned}
\sum_{i=0}^{\infty} 2 \varepsilon_{i} x^{i} & +\sum_{i=0}^{\infty} \varepsilon_{i} x^{i+1}+\cdots+\sum_{i=0}^{\infty} \varepsilon_{i} x^{i+7} \\
= & \sum_{i=0}^{\infty} \varepsilon_{i} x^{2 i}-\sum_{i=0}^{\infty} \varepsilon_{i} x^{2 i+8}+\sum_{i=0}^{\infty} x^{i}+\sum_{i=0}^{\infty} x^{i+1}+\sum_{i=0}^{\infty} x^{i+3} \\
& +\sum_{i=0}^{\infty} x^{i+5}+\sum_{i=0}^{\infty} x^{i+7}+\sum_{i=0}^{\infty} x^{16 i+8}+\sum_{i=0}^{16 N+6} \alpha_{i} x^{i}
\end{aligned}
$$

Comparing the coefficients of $x^{16 N+t}, t=7,9,11,13$, $15,17,19,21,23,25$ on the both sides of $(10)$, we have

$$
2 \varepsilon_{16 N+t}+\varepsilon_{16 N+t-1}+\cdots+\varepsilon_{16 N+t-7}=5, \quad t=7,9, \ldots, 25
$$

Then we have

$$
\begin{gathered}
2 \varepsilon_{16 N+7}+\varepsilon_{16 N+6}+\cdots+\varepsilon_{16 N}=5, \\
2 \varepsilon_{16 N+9}=\varepsilon_{16 N+7}+\varepsilon_{16 N+1}, \\
2 \varepsilon_{16 N+11}+\varepsilon_{16 N+10}=\varepsilon_{16 N+9}+\varepsilon_{16 N+3}+\varepsilon_{16 N+2}, \\
2 \varepsilon_{16 N+13}+\varepsilon_{16 N+12}=\varepsilon_{16 N+11}+\varepsilon_{16 N+5}+\varepsilon_{16 N+4}, \\
2 \varepsilon_{16 N+15}+\varepsilon_{16 N+14}=\varepsilon_{16 N+13}+\varepsilon_{16 N+7}+\varepsilon_{16 N+6}, \\
2 \varepsilon_{16 N+17}=\varepsilon_{16 N+15}+\varepsilon_{16 N+9}, \\
2 \varepsilon_{16 N+19}+\varepsilon_{16 N+18}=\varepsilon_{16 N+17}+\varepsilon_{16 N+11}+\varepsilon_{16 N+10}, \\
2 \varepsilon_{16 N+21}+\varepsilon_{16 N+20}=\varepsilon_{16 N+19}+\varepsilon_{16 N+13}+\varepsilon_{16 N+12}, \\
2 \varepsilon_{16 N+23}+\varepsilon_{16 N+22}=\varepsilon_{16 N+21}+\varepsilon_{16 N+15}+\varepsilon_{16 N+14}, \\
2 \varepsilon_{16 N+25}=\varepsilon_{16 N+23}+\varepsilon_{16 N+17} .
\end{gathered}
$$

Since $A \cap B=\{8 k: k \in \mathbb{N}\}$, we have $\varepsilon_{16 N+m}=1$, $m=0,8,16,24$. Then, by (12), $\left(\varepsilon_{16 N+1}, \varepsilon_{16 N+2}\right.$, 
$\left.\varepsilon_{16 N+3}, \varepsilon_{16 N+4}, \varepsilon_{16 N+5}, \varepsilon_{16 N+6}, \varepsilon_{16 N+7}\right)$ has the following 30 cases:

$$
\begin{aligned}
& (0,0,0,0,1,1,1),(0,0,0,1,0,1,1),(0,0,0,1,1,0,1), \\
& (0,0,1,0,0,1,1),(0,0,1,0,1,0,1),(0,0,1,1,0,0,1), \\
& (0,0,1,1,1,1,0),(0,1,0,0,0,1,1),(0,1,0,0,1,0,1), \\
& (0,1,0,1,0,0,1),(0,1,0,1,1,1,0),(0,1,1,0,0,0,1), \\
& (0,1,1,0,1,1,0),(0,1,1,1,0,1,0),(0,1,1,1,1,0,0), \\
& (1,0,0,0,0,1,1),(1,0,0,0,1,0,1),(1,0,0,1,0,0,1), \\
& (1,0,0,1,1,1,0),(1,0,1,0,0,0,1),(1,0,1,0,1,1,0), \\
& (1,0,1,1,0,1,0),(1,0,1,1,1,0,0),(1,1,0,0,0,0,1), \\
& (1,1,0,0,1,1,0),(1,1,0,1,0,1,0),(1,1,0,1,1,0,0), \\
& (1,1,1,0,0,1,0),(1,1,1,0,1,0,0),(1,1,1,1,0,0,0) .
\end{aligned}
$$

By (13), we have

$$
\varepsilon_{16 N+9}=\varepsilon_{16 N+7}=\varepsilon_{16 N+1}=0,
$$

or

$$
\varepsilon_{16 N+9}=\varepsilon_{16 N+7}=\varepsilon_{16 N+1}=1 \text {. }
$$

Thus $\left(\varepsilon_{16 N+2}, \varepsilon_{16 N+3}, \varepsilon_{16 N+4}, \varepsilon_{16 N+5}, \varepsilon_{16 N+6}, \varepsilon_{16 N+7}, \varepsilon_{16 N+9}\right)$ has the following 10 cases:

$$
\begin{aligned}
& (0,1,1,1,1,0,0),(1,0,1,1,1,0,0),(1,1,0,1,1,0,0), \\
& (1,1,1,0,1,0,0),(1,1,1,1,0,0,0),(0,0,0,0,1,1,1), \\
& (0,0,0,1,0,1,1),(0,0,1,0,0,1,1),(0,1,0,0,0,1,1), \\
& (1,0,0,0,0,1,1) .
\end{aligned}
$$

By (14) and (25), we have $\left(\varepsilon_{16 N+4}, \varepsilon_{16 N+5}, \varepsilon_{16 N+6}\right.$, $\left.\varepsilon_{16 N+7}, \varepsilon_{16 N+9}, \varepsilon_{16 N+10}, \varepsilon_{16 N+11}\right)$ which has the following 8 cases:

$$
\begin{aligned}
& (1,1,1,0,0,1,0),(0,1,1,0,0,0,1),(1,0,1,0,0,0,1), \\
& (1,1,0,0,0,0,1),(0,0,1,1,1,1,0),(0,1,0,1,1,1,0), \\
& (1,0,0,1,1,1,0),(0,0,0,1,1,0,1) .
\end{aligned}
$$

By (15) and (26), we have $\left(\varepsilon_{16 N+6}, \varepsilon_{16 N+7}, \varepsilon_{16 N+9}, \varepsilon_{16 N+10}\right.$, $\left.\varepsilon_{16 N+11}, \varepsilon_{16 N+12}, \varepsilon_{16 N+13}\right)$ which has the following 6 cases:

$$
\begin{aligned}
& (1,0,0,1,0,0,1),(1,0,0,0,1,0,1),(0,0,0,0,1,1,1), \\
& (1,1,1,1,0,0,0),(0,1,1,1,0,1,0),(0,1,1,0,1,1,0) .
\end{aligned}
$$

By (16) and (27), we have $\left(\varepsilon_{16 N+9}, \varepsilon_{16 N+10}, \varepsilon_{16 N+11}, \varepsilon_{16 N+12}\right.$, $\left.\varepsilon_{16 N+13}, \varepsilon_{16 N+14}, \varepsilon_{16 N+15}\right)$ which has the following 6 cases:

$$
\begin{aligned}
& (0,1,0,0,1,0,1),(0,0,1,0,1,0,1),(0,0,1,1,1,1,0), \\
& (1,1,0,0,0,0,1),(1,1,0,1,0,1,0),(1,0,1,1,0,1,0) .
\end{aligned}
$$

By (17) and (28), we have $\left(\varepsilon_{16 N+10}, \varepsilon_{16 N+11}, \varepsilon_{16 N+12}, \varepsilon_{16 N+13}\right.$, $\left.\varepsilon_{16 N+14}, \varepsilon_{16 N+15}, \varepsilon_{16 N+17}\right)$ which has the following 2 cases:

$$
(0,1,1,1,1,0,0),(1,0,0,0,0,1,1) .
$$

By (18) and (29), we have $\left(\varepsilon_{16 N+12}, \varepsilon_{16 N+13}, \varepsilon_{16 N+14}, \varepsilon_{16 N+15}\right.$, $\left.\varepsilon_{16 N+17}, \varepsilon_{16 N+18}, \varepsilon_{16 N+19}\right)$ which has the following 2 cases:

$$
(1,1,1,0,0,1,0),(0,0,0,1,1,0,1) \text {. }
$$

By (19) and (30), we have $\left(\varepsilon_{16 N+14}, \varepsilon_{16 N+15}, \varepsilon_{16 N+17}, \varepsilon_{16 N+18}\right.$, $\left.\varepsilon_{16 N+19}, \varepsilon_{16 N+20}, \varepsilon_{16 N+21}\right)$ which has the following 2 cases:

$$
(1,0,0,1,0,0,1),(0,1,1,0,1,1,0) .
$$

By (20) and (31), we have $\left(\varepsilon_{16 N+17}, \varepsilon_{16 N+18}, \varepsilon_{16 N+19}, \varepsilon_{16 N+20}\right.$, $\left.\varepsilon_{16 N+21}, \varepsilon_{16 N+22}, \varepsilon_{16 N+23}\right)$ which has the following 2 cases:

$$
(0,1,0,0,1,0,1),(1,0,1,1,0,1,0) \text {. }
$$

By (21), we have

$$
\varepsilon_{16 N+25}=\varepsilon_{16 N+23}=\varepsilon_{16 N+17}=0,
$$

or

$$
\varepsilon_{16 N+25}=\varepsilon_{16 N+23}=\varepsilon_{16 N+17}=1,
$$

which contradicts (32).

This completes the proof of Theorem 1.

\section{Proof of Theorem 2}

Proof. Suppose that there exist integer $n_{0}$ and sets $A, B \subset \mathbb{N}$ with $A \cup B=\mathbb{N}$ and $A \cap B=\{8 k: k \in \mathbb{N}\}$ such that $R_{3}(A, n)=$ $R_{3}(B, n)$ for all $n \geq n_{0}$. Without loss of generality, we may assume that $n_{0}=16 N-1, N$ is a positive integer then there exists a polynomial $q(x)$ of degree at most $16 N-2$ such that

$$
\sum_{n=0}^{\infty}\left(R_{3}(A, n)-R_{3}(B, n)\right) x^{n}=q(x) .
$$

Define $f(x), g(x)$, and $\varepsilon_{i}$ as in the proof of Theorem 1 . Then we have

$$
\sum_{n=0}^{\infty} R_{3}(A, n) x^{n}=\frac{1}{2}\left(f^{2}(x)+f\left(x^{2}\right)\right),
$$

$$
\begin{aligned}
\sum_{n=0}^{\infty} R_{3}(B, n) x^{n}= & \frac{1}{2}\left(g^{2}(x)+g\left(x^{2}\right)\right) \\
= & \frac{1}{2}\left(\left(\frac{1}{1-x}+\frac{1}{1-x^{8}}-f(x)\right)^{2}\right. \\
& \left.\quad+\left(\frac{1}{1-x^{2}}+\frac{1}{1-x^{16}}-f\left(x^{2}\right)\right)\right) \\
= & \frac{1}{(1-x)\left(1-x^{2}\right)}+\frac{1}{\left(1-x^{8}\right)\left(1-x^{16}\right)} \\
& +\frac{1}{(1-x)\left(1-x^{8}\right)}-\frac{f(x)}{1-x}-\frac{f(x)}{1-x^{8}} \\
& +\frac{1}{2} f^{2}(x)-\frac{1}{2} f\left(x^{2}\right) .
\end{aligned}
$$


Therefore,

$$
\begin{aligned}
\sum_{n=0}^{\infty}\left(R_{3}(A, n)-R_{3}(B, n)\right) x^{n} \\
=\frac{f(x)}{1-x}+\frac{f(x)}{1-x^{8}}+f\left(x^{2}\right)-\frac{1}{(1-x)\left(1-x^{2}\right)} \\
\quad-\frac{1}{\left(1-x^{8}\right)\left(1-x^{16}\right)}-\frac{1}{(1-x)\left(1-x^{8}\right)} .
\end{aligned}
$$

Write

$$
q(x)\left(1-x^{8}\right)=\sum_{i=0}^{16 N+6} \beta_{i} x^{i} .
$$

By (35) and (37), we have

$$
\begin{aligned}
\left(\sum_{i=0}^{7} x^{i}+1\right) f(x)= & \left(x^{8}-1\right) f\left(x^{2}\right) \\
& +\frac{2+x^{2}+x^{4}+x^{6}}{1-x} \\
& +\frac{1}{1-x^{16}}+q(x)\left(1-x^{8}\right) .
\end{aligned}
$$

We write both sides of (39) in power series; then we have

$$
\begin{aligned}
\sum_{i=0}^{\infty} 2 \varepsilon_{i} x^{i} & +\sum_{i=0}^{\infty} \varepsilon_{i} x^{i+1}+\cdots+\sum_{i=0}^{\infty} \varepsilon_{i} x^{i+7} \\
= & \sum_{i=0}^{\infty} \varepsilon_{i} x^{2 i+8}-\sum_{i=0}^{\infty} \varepsilon_{i} x^{2 i}+\sum_{i=0}^{\infty} 2 x^{i}+\sum_{i=0}^{\infty} x^{i+2} \\
& +\sum_{i=0}^{\infty} x^{i+4}+\sum_{i=0}^{\infty} x^{i+6}+\sum_{i=0}^{\infty} x^{16 i}+\sum_{i=0}^{16 N+6} \beta_{i} x^{i} .
\end{aligned}
$$

Comparing the coefficients of $x^{16 N+t}, t=7,9,11,13,15$, $17,19,21,23,25$ on the both sides of (40), we have

$$
2 \varepsilon_{16 N+t}+\varepsilon_{16 N+t-1}+\cdots+\varepsilon_{16 N+t-7}=5, \quad t=7,9, \ldots, 25 .
$$

The remainder of the proof is the same as that of the proof of Theorem 1 . We omit it here.

\section{Acknowledgments}

This work was supported by the National Natural Science Foundation of China, Grant no. 10901002 and Anhui Provincial Natural Science Foundation, Grant no. 1208085QA02.

\section{References}

[1] G. Dombi, "Additive properties of certain sets," Acta Arithmetica, vol. 103, no. 2, pp. 137-146, 2002.

[2] Y. G. Chen and B. Wang, "On additive properties of two special sequences," Acta Arithmetica, vol. 110, no. 3, pp. 299-303, 2003.
[3] Y. G. Chen, "On the values of representation functions," Science China Mathematics, vol. 54, no. 7, pp. 1317-1331, 2011.

[4] Y. G. Chen and M. Tang, "Partitions of natural numbers with the same representation functions," Journal of Number Theory, vol. 129, no. 11, pp. 2689-2695, 2009.

[5] V. F. Lev, "Reconstructing integer sets from their representation functions," Electronic Journal of Combinatorics, vol. 11, article R78, 2004.

[6] C. Sándor, "Partitions of natural numbers and their representation functions," Integers, vol. 4, article A18, 2004.

[7] M. Tang, "Partitions of the set of natural numbers and their representation functions," Discrete Mathematics, vol. 308, pp. 2614-2616, 2008.

[8] W. Yu and M. Tang, "A note on partitions of natural numbers and their representation functions," preprint. 


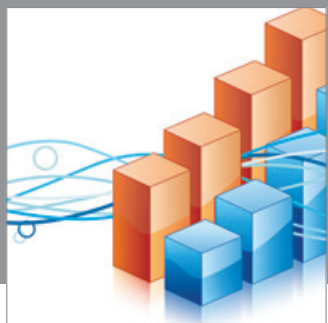

Advances in

Operations Research

mansans

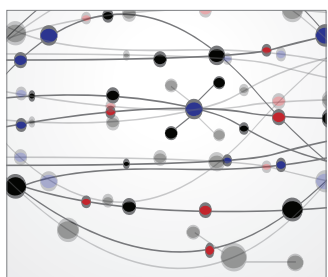

The Scientific World Journal
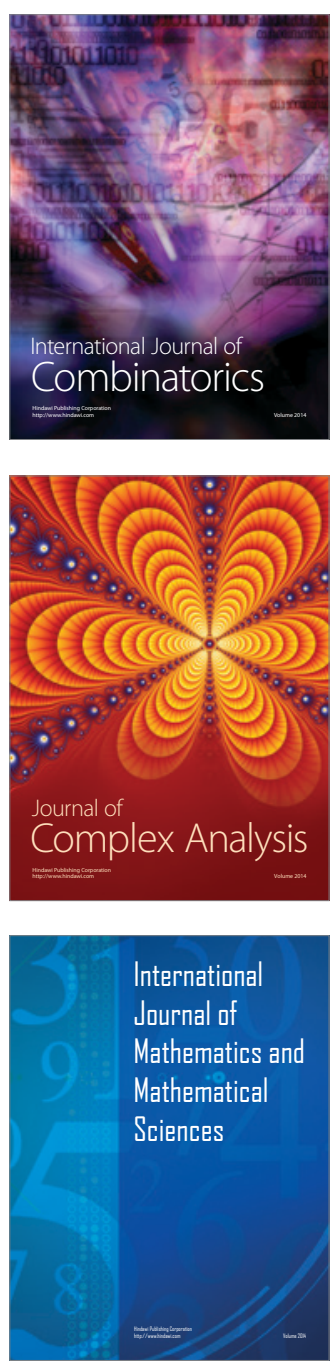
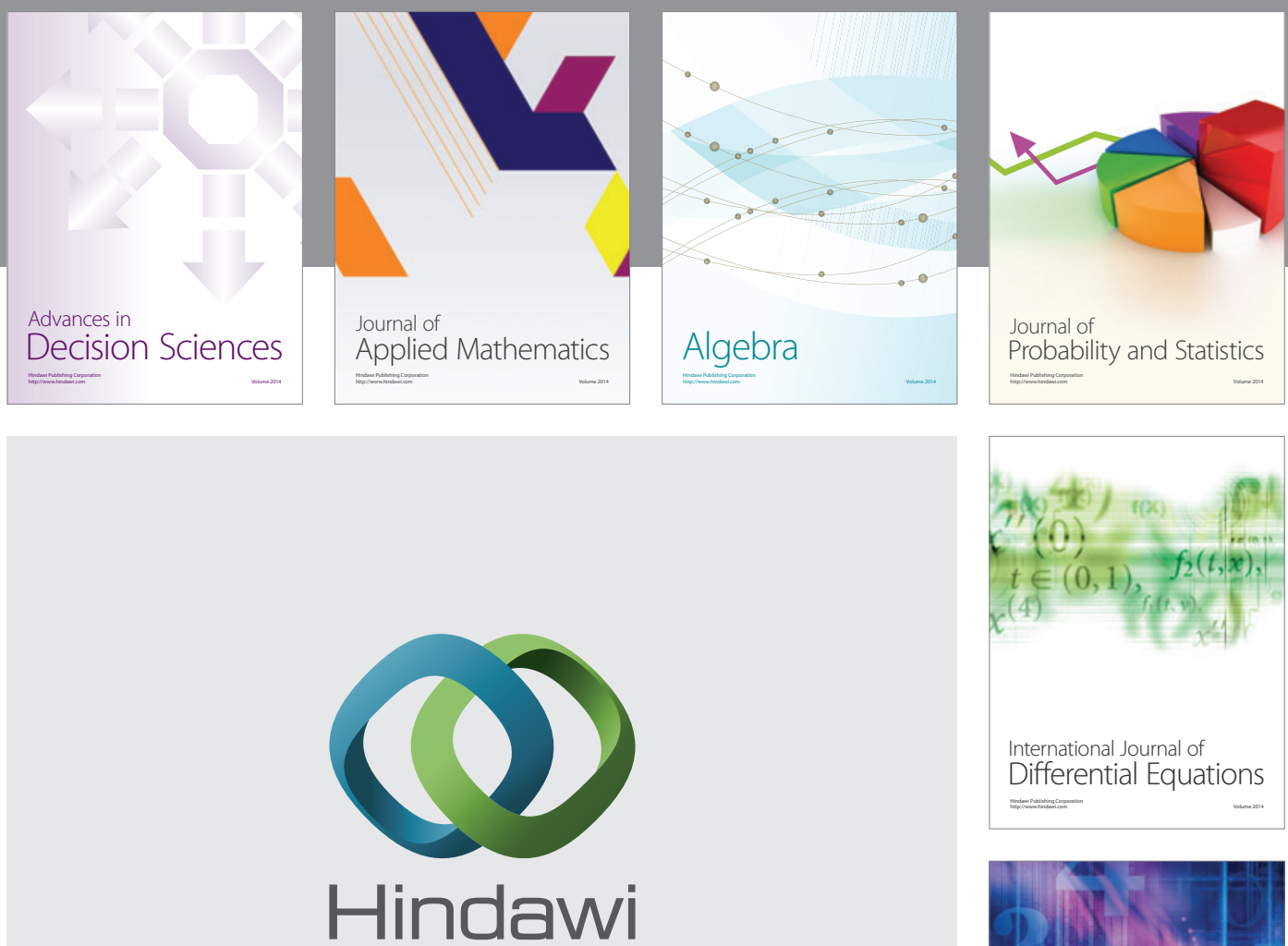

Submit your manuscripts at http://www.hindawi.com
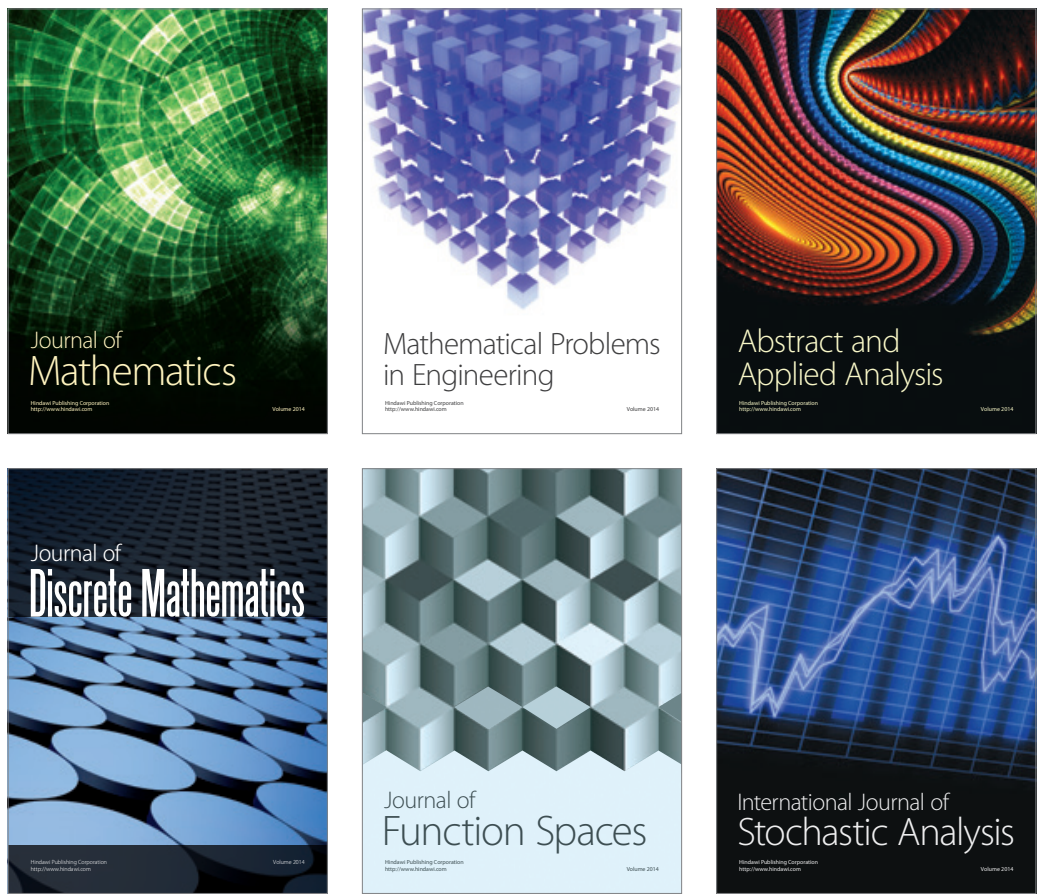

Journal of

Function Spaces

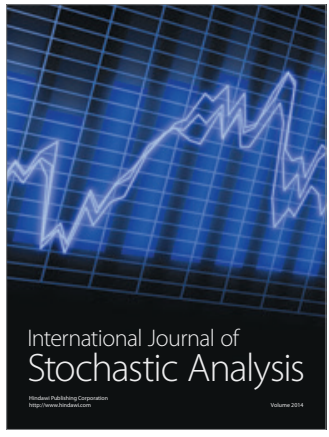

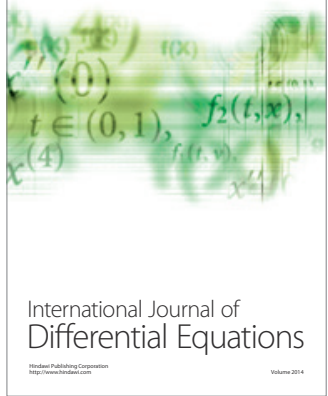
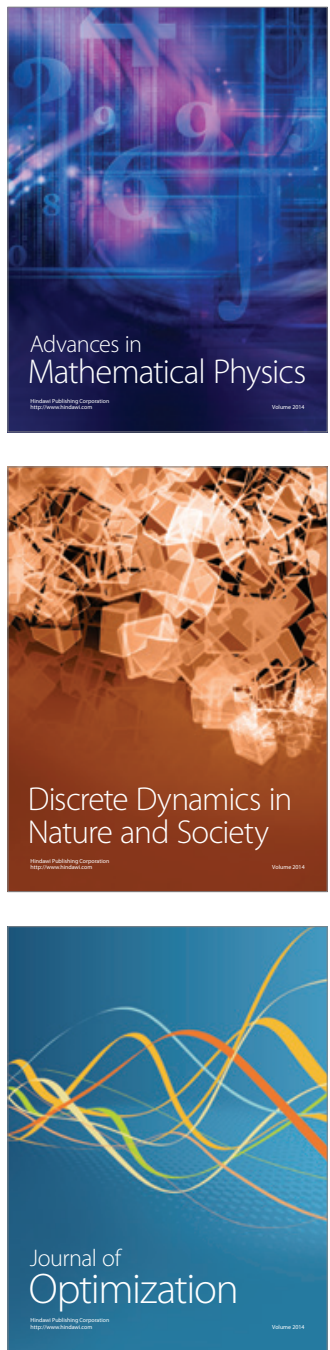